\title{
Mars Cruise Orbit Determination from Combined Optical Celestial Techniques and X-ray Pulsars
}

\author{
Jiandong Liu ${ }^{1,2}$, Erhu Wei ${ }^{3}$ and Shuanggen Jin ${ }^{1,4}$ \\ ${ }^{1}$ (Shanghai Astronomical Observatory, Chinese Academy of Sciences, \\ Shanghai 200030, China) \\ ${ }^{2}$ (University of Chinese Academy of Sciences, Beijing 100049, China) \\ ${ }^{3}$ (School of Geodesy and Geomatics, Wuhan University, Wuhan 430079, China) \\ ${ }^{4}$ (Department of Geomatics Engineering, Bulent Ecevity University, \\ Zonguldak 67100, Turkey) \\ (E-mail: ehwei@sgg.whu.edu.cn)
}

\begin{abstract}
The precise autonomous navigation for deep space exploration by combination of multi-source observation data is a key issue for probe control and scientific applications. In this paper, the performance of an integrated Optical Celestial Navigation (OCN) and X-ray Pulsars Autonomous Navigation (XNAV) system is investigated for the orbit of Mars Pathfinder. Firstly, OCN and XNAV single systems are realised by an Unscented Kalman Filter (UKF). Secondly, the integrated system is simulated with a Federated Kalman Filter (FKF), which can do the information fusion of the two subsystems of UKF and inherits the advantages of each subsystem. Thirdly, the performance of our system is evaluated by analysing the relationship between observation errors and navigation accuracy. The results of the simulation experiments show that the biases between the nominal and our calculated orbit are within $5 \mathrm{~km}$ in all three axes under complex error conditions. This accuracy is also better than current ground-based techniques.
\end{abstract}

\section{KEYW ORDS}
1. Autonomous Navigation.
2. Optical Celestial Navigation.
4. X-ray Pulsars Navigation.
3. Mars Spacecraft Orbit.

Submitted: 31 August 2016. Accepted: 19 November 2016. First published online: 6 February 2017.

1. INTRODUCTION. One of the most important factors for deep space exploration is the positioning and navigation ability of the artificial spacecraft (Jin et al., 2013; Jin and Zhang, 2014). However, current deep space navigation methods strongly depend on ground observations of radiometric tracking data, such as Very Long Baseline Interferometry (VLBI) (Wei et al., 2016) and Unified S-Band (USB) (Bhaskaran, 2012). One of these ground-tracking strategies is the Deep Space Network (DSN) of the United States, which 
has successfully completed most of the deep space missions' navigational work. Nevertheless, with the complicated execution of the system, the errors grow with increasing distance and the resulting real-time navigation and telecommunication problems are all disadvantages of this network (Hemmati, 2006; Graven et al., 2008). Thus, numerous autonomous navigation and integrated navigation methods are proposed to resolve the above problems.

New autonomous methods such as Optical Celestial Navigation (OCN) and X-ray Pulsars Navigation (XNAV) have been mostly focused on deep space navigation since the 1970s. OCN was initially tested and applied densely in low orbit satellite autonomous orbit determination (Gounley et al., 1984; White et al., 1985), in which the orbit accuracy is from several hundred metres to several kilometres. Recently, OCN techniques have been utilised in deep space exploration missions, for example, Deep Space 1, Deep Impact, Mars Reconnaissance Orbiter (MRO) and The Dawn (Soderblom et al., 2002; Hampton et al., 2005; Graf et al., 2005; Mastrodemos et al., 2005; Sierks et al., 2011). The navigation accuracy of OCN for these missions varies between several tens of kilometres and a few hundreds of kilometres. An orbit uncertainty of this magnitude satisfies the requirement for stable navigational and engineered trajectory determination, but cannot fulfil the accuracy demand of scientific applications.

Downs (1974) first proposed the idea of using celestial X-ray sources for interplanetary navigation. Chester and Butman (1981) utilised X-ray Pulsars for navigation simulation, and an accuracy of around 150 kilometres can be achieved based on their theory. Recent simulation results have shown that the navigation accuracy of this method could reach within five kilometres (Sheikh et al., 2006; Becker et al., 2013; Deng et al., 2013). For example, simulations using XNAV to determine spacecraft orbits around Mars show that the accuracy of this method is within two kilometres (Wei et al., 2013). In addition, several testing plans and XNAV navigation experiments have been done or will be done, including Unconventional Stellar Aspect (USA), X-ray Source-based Navigation for Autonomous Position Determination (XSNAPD), and the Station Explorer for X-ray Timing and Navigation Technology (SEXTANT) (Hanson, 2006; Sheikh and Pines, 2006; Winternitz et al., 2016). These experiments have all confirmed the feasibility of XNAV. In particular the SEXTANT ground test experiments demonstrated that the accuracy of this system could achieve an orbit accuracy of ten kilometres with current hardware configurations.

Currently, the accuracy of the time delay of VLBI is of a magnitude of nanoseconds, which equals to range errors of about $4 \mathrm{~km}$ per Astronomical Unit (AU) (James et al., 2009; Becker et al., 2013; Cao et al., 2010). The OCN used on deep space spacecraft for autonomous navigation purposes has far poorer accuracy than the ground-based techniques. At the same time, the signal intensity of the XNAV is so weak that the observations have to be folded and integrated over several minutes to several tens of minutes according to the flux of the X-ray Pulsars (Sheikh et al., 2006; Becker et al., 2013). Thus, the performance of this system is not stable and the system would suffer collapse in some cases. Accordingly, an integrated navigation system of OCN/XNAV would be a better choice for future deep space navigation.

Many integrated system and filter designs of OCN/XNAV have been simulated to calculate Earth satellite orbits and orbits circling around Mars (Wang et al., 2013; Rong et al., 2016). The current simulated accuracy of XNAV or XNAV/OCN that achieved an error magnitude of several kilometres is available, however, the techniques which achieved hundred metres accuracy would generate hardware requirements that would currently be 
difficult to fulfil according to the ground-testing experiments of SEXTANT. These simulations are more concerned about either the performance of the Kalman filter or the design of the filter. Most of the simulations make minor consideration of the influence of complex errors on the performance of integrated OCN/XNAV.

In this paper, the OCN/XNAV system is realized and evaluated by simulation. The spacecraft's orbit accuracy is evaluated in many different conditions such as sensors' pointing accuracy, the integration time of the XNAV, the delay errors and the clock errors. The orbit uncertainty is studied and analysed to give an autonomous, stable and high accuracy orbit solution. The paper is organised as follows: the principle of the OCN/XNAV integrated navigation system is presented in Section 2. A numerical experiment is demonstrated in Section 3 to test the accuracy and robustness of the system. The relationship between system parameters and the accuracy of the orbit is also discussed and analysed in this section. Finally, conclusions are given in Section 4.

\section{METHODS AND MODELS.}

2.1. Dynamics model of the Mars spacecraft. Satellite Tool Kit (STK) software is utilised to generate the nominal orbit, which has the same condition and orbit manoeuvre as the Mars Pathfinder spacecraft. It should be noted that all our accuracy evaluation and observation simulation is based on this nominal orbit. Since our paper is focused on evaluating the performance of the integrated OCN/XNAV navigation system, we use a cyclo-restricted four-body model concerning the Sun, Mars, the Earth and the spacecraft to build the dynamics model to simplify the problem. Thus, the accelerated speed $\boldsymbol{a}$ of the spacecraft in the cruise phase can be expressed as:

$$
\mathbf{a}=-\frac{\mu_{s}}{r_{p s}^{3}} \mathbf{r}_{p s}-\mathbf{a}_{m}-\mathbf{a}_{e}+\omega
$$

where $\mu_{s}$ represents the heliocentric gravitational constant; $\mathbf{r}_{p s}$ represents the position and velocity vectors of the spacecraft in the J2000 Heliocentric Inertial Coordinate System (J2000HICS) that is defined by DE405; $\boldsymbol{r}_{p s}=\left[\begin{array}{llllll}x & y & z & \dot{x} & \dot{y} & \dot{z}\end{array}\right]^{T}, r_{p s}=\sqrt{x^{2}+y^{2}+z^{2}}, \boldsymbol{a}=\dot{\boldsymbol{r}}_{p s}$. The dot above the variable means its derivative; $\boldsymbol{a}_{m}$ and $\boldsymbol{a}_{e}$ represent the gravitational attraction of Mars and the Earth to the spacecraft, respectively. $\omega$ is the accelerated speed noise. The perturbative force of Mars and the Earth is expressed as:

$$
\left\{\begin{array}{l}
\boldsymbol{a}_{m}=\frac{\mu_{m}}{r_{p m}^{3}} \boldsymbol{r}_{p m}-\frac{\mu_{m}}{r_{s m}^{3}} \boldsymbol{r}_{s m} \\
\boldsymbol{a}_{e}=\frac{\mu_{e}}{r_{p e}^{3}} \boldsymbol{r}_{p e}-\frac{\mu_{e}}{r_{s e}^{3}} \boldsymbol{r}_{s e}
\end{array}\right.
$$

where, $\mu_{m}$ and $\mu_{e}$ represent Mars-centric and geocentric gravitational constants, $\boldsymbol{r}_{p m}$ and $\boldsymbol{r}_{p e}$ represent the position and velocity of the spacecraft relative to Mars and Earth, $\boldsymbol{r}_{s m}$ and $\boldsymbol{r}_{s e}$ are Mars and Earth position and velocity vectors in J2000HICS which can be acquired from DE405. $r_{p m}, r_{p e}, r_{s m}$ and $r_{s e}$ are the distances of the above vectors which have similar formulae to $r_{p s}$.

2.2. Algorithm of the integrated navigation system.

2.2.1. Numeric solution of the dynamic equation. In this paper, an Unscented Kalman Filter (UKF) is utilised to conduct orbit calculation to avoid linearizing Equation (1), the $\mathrm{OCN}$ observation equation and the XNAV observation equation. 
Table 1. SLDA simulated in this paper.

\begin{tabular}{ccccc}
\hline Angles & Sun-sensor-Sirius & Sun-sensor-Earth & Earth-sensor-Mars & Sun-sensor-Mars \\
\hline Name & $\theta$ & $\phi$ & $\varphi$ & $\psi$ \\
\hline
\end{tabular}

Table 2. Pointing accuracy of the celestial sensors.

\begin{tabular}{lcccc}
\hline Sensors & Star Sensor & Sun Sensor & Earth Sensor & Mars Sensor \\
\hline High accuracy & $1^{\prime \prime}$ & $1^{\prime \prime}$ & $0 \cdot 001^{\circ}$ & $0.001^{\circ}$ \\
Medium accuracy & $2^{\prime \prime}$ & $3^{\prime \prime}$ & $0.002^{\circ}$ & $0.002^{\circ}$ \\
& $3^{\prime \prime}$ & $5^{\prime \prime}$ & $0.003^{\circ}$ & $0.003^{\circ}$ \\
Low accuracy & $4^{\prime \prime}$ & $6^{\prime \prime}$ & $0.004^{\circ}$ & $0.004^{\circ}$ \\
& $5^{\prime \prime}$ & $7^{\prime \prime}$ & $0.005^{\circ}$ & $0.005^{\circ}$ \\
& $6^{\prime \prime}$ & $8^{\prime \prime}$ & $0.005^{\circ}$ & $0.005^{\circ}$ \\
\hline
\end{tabular}

Equation (1) can be solved by numerical integration method. Therefore, we use RungeKutta (RK) methods in terms of RK 7(8) to acquire the initial solution that can achieve enough solution accuracy (Dormand and Prince, 1978). This solution also provides the initial Sigma points (Section 2.2.4).

2.2.2. Measurement model of OCN. Particularly, our paper focuses on one of the observational methods of OCN that is called Stellar Light Distance Angle (SLDA) because this observation has been put into use in several deep space spacecraft navigations (Lowman and Stauder, 2004). SLDA is the angle between two light rays of celestial bodies observed from sensors such as navigation camera on board the spacecraft. We simulate four angles that are illustrated in Table 1.

The simulation process is as follows: First, the SLDA can be deduced from the unit direction vector of the stellar light as Equation (3) shows,

$$
Z_{l}=\arccos \left(\boldsymbol{l}_{p 1} \cdot \boldsymbol{l}_{p 2}\right)+\boldsymbol{v}_{\boldsymbol{l}}
$$

where $\boldsymbol{Z}_{\boldsymbol{l}}=\left[\begin{array}{llll}\theta & \phi & \varphi & \psi\end{array}\right]^{T}$ is the observation vector, $\boldsymbol{l}_{p 1}=\left[\begin{array}{llll}\boldsymbol{l}_{\boldsymbol{p} \boldsymbol{b}} & \boldsymbol{l}_{\boldsymbol{p s}} & \boldsymbol{l}_{\boldsymbol{p e}} & \boldsymbol{l}_{\boldsymbol{p s}}\end{array}\right]^{T}$ and $\boldsymbol{l}_{p 2}=$ $\left[\begin{array}{llll}\boldsymbol{l}_{\boldsymbol{p} s} & \boldsymbol{l}_{\boldsymbol{p}} & \boldsymbol{l}_{\boldsymbol{p} m} & \boldsymbol{l}_{\boldsymbol{p} m}\end{array}\right]^{T}$ are stellar unit direction vectors in which the subscripts $p, b, s, e$ and $m$ represents the centre of the spacecraft, celestial body Sirius, the Sun, the Earth and Mars, respectively. Accordingly, the unit vectors can be acquired from radius vectors. $\boldsymbol{v}_{l}$ is the noise vector of the equation. Specifically, these direction vectors are acquired from the nominal orbit and DE405. Secondly, when the true SLDA observation is deduced from Equation (3) (excluding random noise $\boldsymbol{v}_{\boldsymbol{l}}$ ), some random errors are added to the true SLDA based on the accuracy of sensors shown in Table 2 (Mastrodemos et al., 2005; Stastny and Geller, 2008; Sun et al., 2016). The accuracy of the SLDA is evaluated by the square root of sum of squares of two point accuracy.

2.2.3. Measurement model of XNAV. The main idea of XNAV is to obtain time delay observation, which is between the Time Of Arrival (TOA) of the Pulsar's signal to the probe and the TOA of the signal to the origin of a (quasi) inertial space-time reference system. The Solar System Barycenter (SSB) is highly recommended because many theories and observations are based on the system. Nevertheless, the origin of the inertial reference system in this paper is the heliocentre for the sake of convenient simulation. A prior model (a pulse template) can predict the TOA to the origin of the inertial reference system (Sheikh et al., 2007). 
The time delay includes some corrections due to interstellar medium dispersion effect, general relativity effect and on board clock uncertainties $\delta t_{c}$ which can be corrected by prior models (Sheikh et al., 2006; Becker et al., 2013). The total time delay $\tau$ should be written as:

$$
\tau=\frac{1}{c} \boldsymbol{n} \cdot \boldsymbol{r}_{\boldsymbol{p s}}+\delta t_{\text {roemer }}+\delta t_{\text {shapiro }}+\delta t_{c}+v_{2}
$$

The first term of the right side in the equation is geometry time delay $\tau_{g} . c$ represents the speed of light, $\boldsymbol{n}$ is the direction vector of the Pulsar signal, $\boldsymbol{r}_{\mathbf{s p}}$ is the position vector of the probe, $\delta t_{\text {romer }}$ is the Roemer delay, $\delta t_{\text {shapiro }}$ is the Shapiro delay (Sheikh et al., 2007) and $v_{2}$ represents observation random noise of XNAV. The Roemer delay ${ }^{1}$ and Shapiro delay is written as the following reduced form:

$$
\left\{\begin{array}{l}
\delta t_{\text {reomer }}=\frac{1}{2 c D_{0}}\left[\left(\mathbf{n} \cdot \mathbf{r}_{\mathbf{p s}}\right)^{2}-r_{p s}^{2}+2(\mathbf{n} \cdot \mathbf{b})\left(\mathbf{n} \cdot \mathbf{r}_{p s}\right)-2\left(\mathbf{b} \cdot \mathbf{r}_{p s}\right)\right] \\
\delta t_{\text {reomer }}=\frac{2 \mu_{s}}{c^{3}} \ln \left|\frac{\mathbf{n} \cdot \mathbf{r}_{p s}+\mathbf{n} \cdot \mathbf{b}+\left\|\mathbf{r}_{p s}+\mathbf{b}\right\|}{\mathbf{n} \cdot \mathbf{b}+b}\right|
\end{array}\right.
$$

$D_{0}$ is the initial position of Pulsars and $\boldsymbol{b}$ is the solar position vector in J2000 BCRS. We use first order clock error model to simulate the performance of the atomic clock on board the spacecraft:

$$
\delta t_{c}=a_{0}+a_{1}\left(t-t_{0}\right)
$$

$a_{0}$ is the clock offset and $a_{1}$ is the clock drift.

To simulate the XNAV observation, firstly, geometry time delay $\tau_{g}$ is deduced from the first term of Equation (4) based on nominal orbit and DE405. In addition, the total time delay is simulated from Equation (4) by adding the three model errors and noise. The accuracy of X-ray Pulsars is evaluated based on the theory introduced by Becker et al. (2013) and can be found in Wei et al. (2013).

2.2.4. The Kalman Filter. As illustrated as above, we use a UKF as the filter algorithm to process the OCN and XNAV observation data. The Unscented Kalman Filter was utilised to estimate nonlinear state in terms of Sigma Points (SP). In this paper, a symmetric sampling method is adopted to generate SP and the standard UKF algorithm is described by Wan and Van der Merwe (2000).

There are three kinds of parameter in terms of scale parameter $\kappa$, SP distribution parameter $\alpha$ and distribution function parameter $v$ that have influence on the performance of UKF (Wan and Van der Merwe, 2000). We adopt $v=2, \kappa=0$ and $\alpha=0 \cdot 1$. Hundreds of experiments have been done to determine these values. The experiments show that the value of $v$ and $\kappa$ have very slight influence on our UKF. However, the accuracy that the calculated trajectory obtained varies depending on the values of $\alpha$ from $10^{-4}$ to 1 . The closer the value is to 1 , the higher the accuracy that the calculated orbit can achieve (evaluated by the bias between nominal orbit and our calculated orbit). The orbit accuracy no longer decreases or increases drastically when $\alpha$ is around $0 \cdot 1$.

Our integrated navigation system is realised by a Federal Kalman Filter (FKF) which was first introduced by Carlson (1990). The main idea of FKF is to obtain a global optimal

\footnotetext{
${ }^{1}$ Actually, the Roemer Delay includes first two terms of right side of Equation (4). Because the first term is utilised to calculate the orbit we regard the second term as a systematic error, which would have influence on the integrated navigation system.
} 


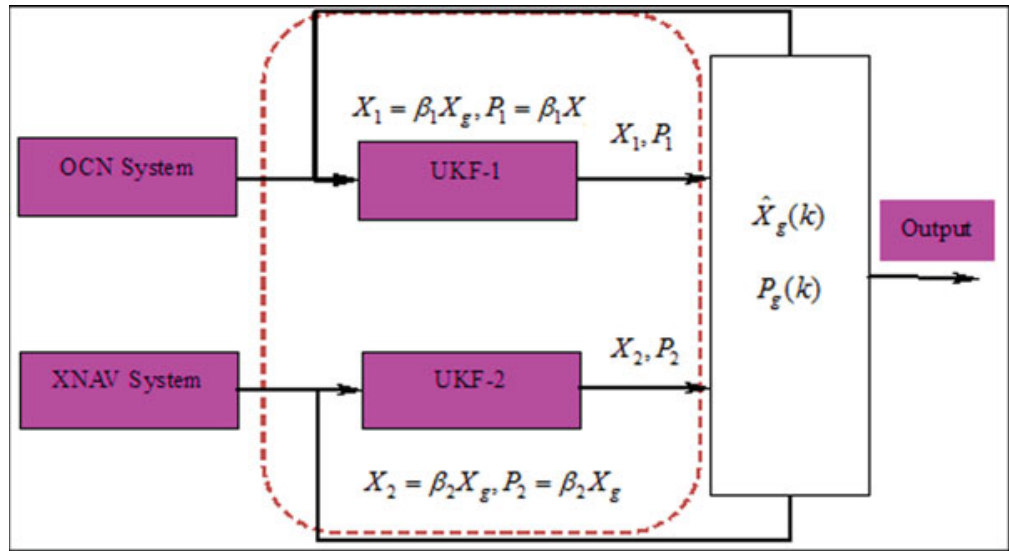

Figure 1. A simplified flow chart of Federal Kalman Filter.

Table 3. The timeline of orbit manoeuvrings of Mars Pathfinder.

\begin{tabular}{lccccc}
\hline The corrections & 1st & 2nd & 3 rd & 4 th & 5 th \\
\hline Time & $97-01-09$ & $97-02-03$ & $97-05-06$ & $97-06-25$ & $97-07-03(4)$
\end{tabular}

solution via merging solutions of subsystems according to a weighted principle and, in turn, the merged solution can be distributed to every subsystem in terms of an Information Distribution Factor (IDF) to form a feedback procedure and provide an accurate initial value for the subsystem.

The FKF flow chart utilised by this paper is illustrated in Figure 1. The global optimal solution $\hat{X}_{g}(t)$ (when $t=k$ ) can be obtained by:

$$
\left\{\begin{aligned}
\hat{X}_{g}(k)= & P_{g}(k) \cdot\left[P_{1}^{-1}(k) X_{1}(k)+P_{1}^{-1}(k) X_{2}(k)\right] \\
& P_{g}(k)=\left[P_{1}^{-1}(k)+P_{1}^{-1}(k)\right]^{-1}
\end{aligned}\right.
$$

What is more, the IDF of the feedback procedure is as follows:

$$
\beta_{j}=\frac{\left\|P_{j}(k)\right\|^{-1}}{\sum_{j=1}^{2}\left\|P_{j}(k)\right\|^{-1}}
$$

where $j=1,2 ;\left\|P_{j}(k)\right\|=\sqrt{\sum \operatorname{diag}\left(P_{j}^{T}(k) \cdot P_{j}(k)\right)}$. Equation (7) can integrate the information from each subsystem and the main system will feedback the state by means of the IDF illustrated in Equation (8).

3. SIMULATION RESULTS AND DISCUSSION. The Mars Pathfinder was launched on 4 December 1996 and landed on Mars on 4 July 1997. In the near seven-month cruise phase the ground navigation group utilised VLBI and USB of the DSN to navigate the probe. The probe conducted four orbit manoeuvrings and one end correction (Golombek et al., 1997), shown in Table 3. 


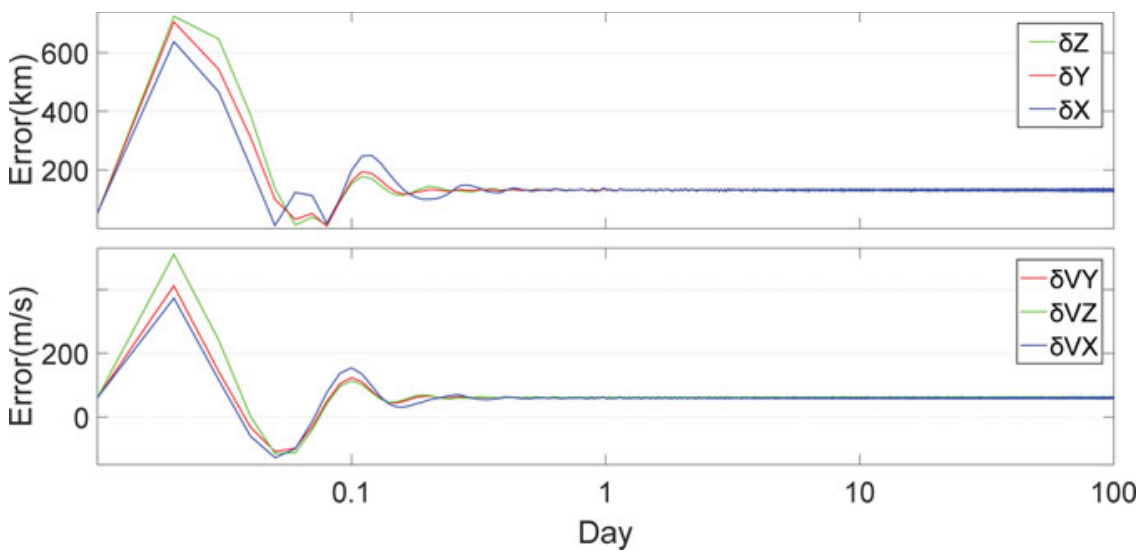

Figure 2. Accuracy of the OCN system. The position and velocity accuracy are evaluated by the biases between calculated orbit and the nominal orbit in this paper.

We use the cruise phase from 1 March 1997 to 9 June 1997 because this timeline includes only one orbit manoeuvring (3rd, 97-05-06). Thus, the orbit would be very stable and is similar to our dynamic model Equation (1). Additionally, 101 days Mars Pathfinder's cruise orbit data are used and the sampling interval is 864 seconds. OCN and XNAV are first tested separately to ensure the validity of our FORTRAN program and to evaluate the performances of the single systems. After that, we use FKF to integrate the single systems and to verify the outstanding nature of the integrated system (main system).

3.1. Single system experiments. The simulated observations of OCN or XNAV are first processed by UKF to evaluate the performance of a single navigation system (OCN or $\mathrm{XNAV}$ ). Particularly, the experiential initial variance matrix of the first step prediction of $\mathrm{OCN}$ and XNAV is as follows:

$$
P(0 \mid 0)=\left[\begin{array}{llllll}
100^{2} \mathrm{~km} & 100^{2} \mathrm{~km} & 100^{2} \mathrm{~km} & 10^{2} \mathrm{~m} / \mathrm{s} & 10^{2} \mathrm{~m} / \mathrm{s} & 10^{2} \mathrm{~m} / \mathrm{s}
\end{array}\right] .
$$

The observation variance of OCN and XNAV is also assumed by the pointing accuracy of the celestial navigation camera and the positioning accuracy of pulsars evaluated by profile integration time, respectively. They are $R_{1}=\left[250^{2} \mathrm{~km} 350^{2} \mathrm{~km} 350^{2} \mathrm{~km} 350^{2} \mathrm{~km}\right]$ for OCN which is equal to the high accuracy navigation camera's precision and $R_{2}=$ $\left[860^{2} \mathrm{~m} 1560^{2} \mathrm{~m} 54^{2} \mathrm{~m} 1493^{2} \mathrm{~m} 932^{2} \mathrm{~m} 282^{2} \mathrm{~m} 151^{2} \mathrm{~m}\right.$ ] for XNAV which is also equal to $500 \mathrm{~s}$ integration time of the pulse profile. The clock error parameters are set as $a_{0}=0 \cdot 1$ nanoseconds and $a_{1}=1$ picoseconds per day. The Roemer Delay and Shapiro Delay are exclusive of this case.

The bias between the calculated trajectory and nominal orbit of OCN is illustrated in Figure $2 . \delta$ represents bias and $X, Y, Z$ are the three axes, respectively. The calculated orbit converges within ten epochs ( 8640 seconds). Moreover, both position and velocity are very stable because the biases after the tenth epoch are very smooth. The accuracy of the three axes is similar to each other and the magnitude of position and velocity errors are a hundred kilometres and several tens of metres per second.

Figure 3 illustrates the calculated orbit determined by XNAV. The biases are symmetric about the zero on all three axes. However, the biases of the position and velocity grow much larger after the 60th day. This phenomenon shows that the orbit has overlap with the 

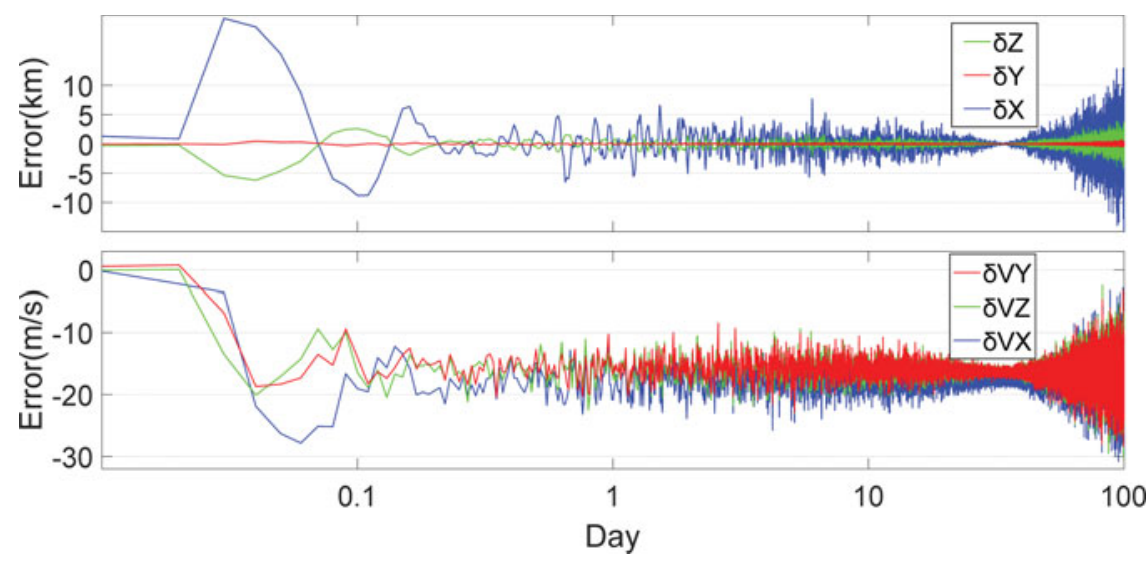

Figure 3. Accuracy of the XNAV system.
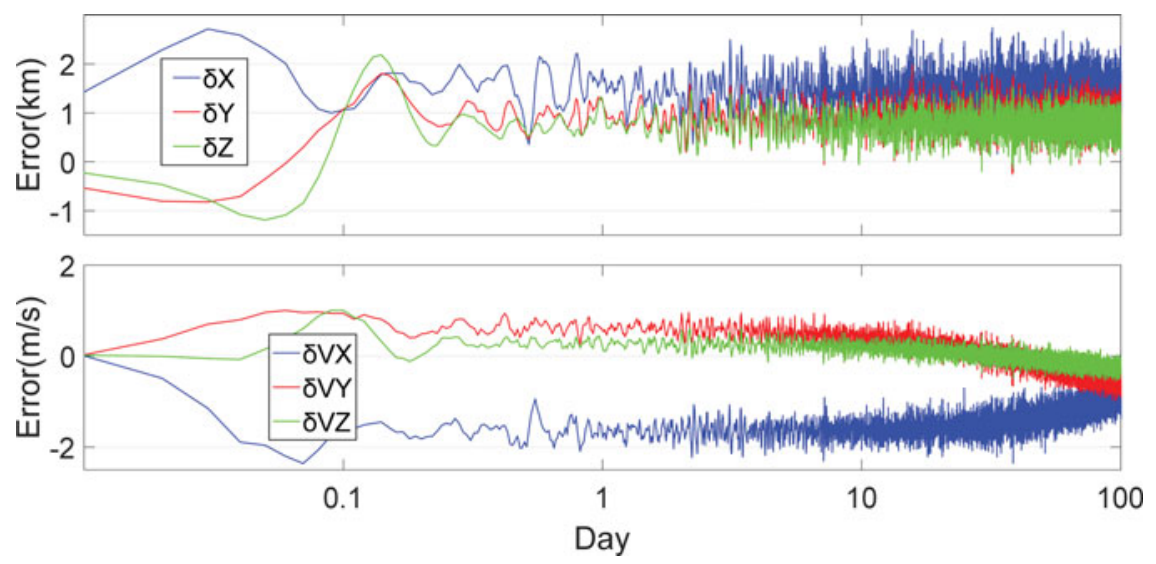

Figure 4. Accuracy of OCN/XNAV integrated navigation system.

nominal orbit, however, the dynamical model might not be so precise at end of the epoch because of the orbit manoeuvring on the 60th day. Moreover, the biases are not similar in the three axes, because the imperfect distribution of navigational X-ray Pulsars (most of them locate in the plane of the galaxy) and because of the nature of the simulated XNAV observations (the geometry of the observations are similar to Space-VLBI).

3.2. Integrated system experiments.

3.2.1. General case. In this general case, the main system is integrated by the above single navigation system via FKF. The parameters such as clock errors are all the same as the single system.

Figure 4 shows that the accuracy of the integrated navigation system is better than single OCN or single XNAV. Additionally, this accuracy is also better than ground VLBI/USB techniques. The bias in the $\mathrm{X}$-axis is a bit larger than the other two axes as shown in Table 4. This result illustrates that the integrated OCN/XNAV inherits both stability and high accuracy from the single navigation system due to the information fusion procedure 
Table 4. Statistical accuracy of integrated system: general case.

\begin{tabular}{lllrl}
\hline Bias & $\max$ & \multicolumn{1}{l}{$\min$} & mean & SD \\
\hline$\delta \mathrm{X} / \mathrm{km}$ & 2.74 & -0.07 & 1.44 & 0.36 \\
$\delta \mathrm{Y} / \mathrm{km}$ & 1.98 & -0.82 & 0.88 & 0.28 \\
$\delta \mathrm{Z} / \mathrm{km}$ & 2.18 & -1.19 & 0.76 & 0.26 \\
$\delta \mathrm{VX} / \mathrm{ms}^{-1}$ & 0.01 & -2.36 & -1.32 & 0.31 \\
$\delta \mathrm{VY} / \mathrm{ms}^{-1}$ & 1.01 & -0.99 & -0.13 & 0.38 \\
$\delta \mathrm{VZ} / \mathrm{ms}^{-1}$ & 1.01 & -0.54 & -0.06 & 0.19 \\
\hline
\end{tabular}

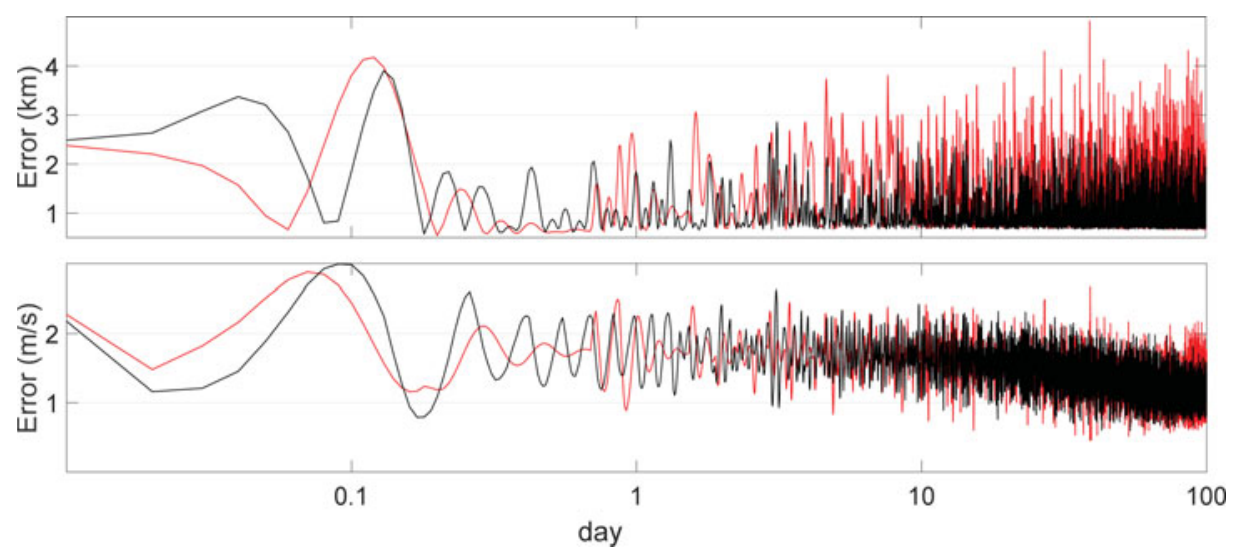

Figure 5. The orbit accuracy with different observation noise: the red line represents the worst orbit accuracy and the black line represents the best orbit accuracy. The orbit accuracy is evaluated by square root of the three axes (Same meaning as Figures 6-9).

provided by FKF. The Standard Deviations (SD) listed in Table 4 show that the integrated system converges well.

3.2.2. Experiments with only random observation errors. In this experiment, we assume that the systematic errors of the integrated system have been eliminated or reduced to the extent that they can be neglected. The pointing accuracy of the celestial sensors in six grades and the integration time of the pulse profile of Pulsars are investigated to evaluate the performance of the system.

Parts of the testing results are illustrated in Figure 5. The statistic results including all of the cases are listed in Table 5 in terms of maximum value, minimum value, mean value and Standard Deviation. The lowest accuracy celestial sensors and shortest integration time that is shown in Table 5 (the 6th case) generate the worst orbit accuracy. The 2 nd to 5 th cases have minor differences to each other in all of the four indices, which indicate that the subOCN systems have very limited accuracy contribution to the main navigation system. The reason will be discussed in Section 3.3. In turn, the accuracy of XNAV observations has a major influence on the integrated system, which can be deduced from the comparison of best case (the 1st case) and the worst case.

3.2.3. Experiments with systematic errors. The Roemer delay and Shapiro delay would have a major influence on the performances of the integrated navigation system because both of these delays are systematic errors. In these experiments, the celestial 
Table 5. The orbit accuracy statistics. The six celestial accuracy grades are shown in Table 2 and the numbers in the parentheses represent integration time of Pulsar profile.

\begin{tabular}{|c|c|c|c|c|c|c|c|c|c|c|c|c|}
\hline \multirow{2}{*}{$\begin{array}{l}\text { Value } \\
\text { Bias }\end{array}$} & \multicolumn{2}{|c|}{$1(1500 \mathrm{~s})$} & \multicolumn{2}{|c|}{$2(1000 \mathrm{~s})$} & \multicolumn{2}{|c|}{$3(1000 \mathrm{~s})$} & \multicolumn{2}{|c|}{$4(1000 s)$} & \multicolumn{2}{|c|}{$5(1000 \mathrm{~s})$} & \multicolumn{2}{|c|}{$6(500 \mathrm{~s})$} \\
\hline & $\begin{array}{l}\max \\
\text { mean }\end{array}$ & $\begin{array}{c}\min \\
\mathrm{SD}\end{array}$ & $\begin{array}{c}\max \\
\text { mean }\end{array}$ & $\begin{array}{c}\min \\
\mathrm{SD}\end{array}$ & $\begin{array}{c}\max \\
\text { mean }\end{array}$ & $\begin{array}{c}\min \\
\mathrm{SD}\end{array}$ & $\begin{array}{c}\max \\
\text { mean }\end{array}$ & $\begin{array}{c}\min \\
\mathrm{SD}\end{array}$ & $\begin{array}{c}\max \\
\text { mean }\end{array}$ & $\begin{array}{c}\min \\
\mathrm{SD}\end{array}$ & $\begin{array}{c}\max \\
\text { mean }\end{array}$ & $\begin{array}{c}\min \\
\mathrm{SD}\end{array}$ \\
\hline \multirow[t]{2}{*}{$\delta \mathrm{X} / \mathrm{km}$} & $3 \cdot 27$ & $-3 \cdot 56$ & $2 \cdot 70$ & $-3 \cdot 84$ & $3 \cdot 18$ & $-3 \cdot 23$ & $2 \cdot 66$ & -2.90 & $2 \cdot 89$ & -2.68 & 3.79 & -4.45 \\
\hline & $-0 \cdot 16$ & 0.73 & $-0 \cdot 18$ & 0.90 & $-0 \cdot 14$ & 0.91 & -0.22 & 0.91 & $-0 \cdot 16$ & 0.92 & $-0 \cdot 19$ & $1 \cdot 28$ \\
\hline \multirow[t]{2}{*}{$\delta \mathrm{Y} / \mathrm{km}$} & 1.07 & 0.21 & 0.94 & $0 \cdot 20$ & 1.07 & 0.24 & 0.94 & 0.26 & 0.97 & $0 \cdot 32$ & 0.78 & $0 \cdot 18$ \\
\hline & 0.48 & 0.06 & 0.48 & 0.08 & 0.48 & 0.08 & 0.47 & 0.08 & 0.48 & 0.08 & 0.47 & $0 \cdot 11$ \\
\hline \multirow[t]{2}{*}{$\delta \mathrm{Z} / \mathrm{km}$} & 1.59 & -0.68 & 1.68 & $-0 \cdot 32$ & 1.46 & -0.63 & 1.50 & $-0 \cdot 34$ & 1.51 & -0.57 & 2.07 & -0.75 \\
\hline & $0 \cdot 60$ & 0.25 & 0.61 & 0.36 & 0.59 & 0.37 & 0.63 & 0.36 & 0.60 & 0.37 & 0.62 & 0.49 \\
\hline \multirow[t]{2}{*}{$\delta \mathrm{r} / \mathrm{km}$} & 3.09 & 0.57 & $4 \cdot 20$ & 0.64 & $3 \cdot 55$ & 0.56 & $3 \cdot 21$ & 0.59 & $3 \cdot 10$ & 0.54 & 4.91 & 0.55 \\
\hline & 1.04 & $0 \cdot 37$ & $1 \cdot 18$ & 0.45 & $1 \cdot 19$ & 0.41 & $1 \cdot 19$ & 0.47 & $1 \cdot 18$ & 0.46 & $1 \cdot 26$ & 0.68 \\
\hline$\delta \mathrm{VX} /$ & $1 \cdot 10$ & $-2 \cdot 85$ & 1.23 & $-2 \cdot 88$ & 1.23 & $-2 \cdot 75$ & $1 \cdot 10$ & -2.69 & 0.99 & -2.45 & 0.83 & -2.73 \\
\hline $\mathrm{ms}^{-1}$ & $-1 \cdot 26$ & 0.37 & $-1 \cdot 26$ & 0.29 & $-1 \cdot 26$ & $0 \cdot 26$ & $-1 \cdot 26$ & 0.29 & $-1 \cdot 26$ & 0.29 & $-1 \cdot 26$ & $0 \cdot 39$ \\
\hline$\delta \mathrm{VY} / \mathrm{ms}^{-1}$ & $\begin{array}{ll}1 & 1.51\end{array}$ & -0.67 & 1.63 & -0.64 & 1.63 & -0.63 & 1.75 & -0.64 & 1.65 & -0.65 & 1.62 & -0.70 \\
\hline & $-0 \cdot 11$ & 0.36 & $-0 \cdot 11$ & $0 \cdot 36$ & $-0 \cdot 11$ & 0.36 & $-0 \cdot 11$ & 0.36 & $-0 \cdot 11$ & 0.36 & $-0 \cdot 11$ & $0 \cdot 36$ \\
\hline$\delta \mathrm{VZ} / \mathrm{ms}^{-1}$ & $\begin{array}{ll}1 & 1 \cdot 12\end{array}$ & -0.63 & $1 \cdot 12$ & -0.59 & 1.24 & -0.66 & $1 \cdot 12$ & -0.60 & 1.25 & -0.61 & $1 \cdot 36$ & -0.92 \\
\hline & -0.07 & $0 \cdot 20$ & -0.07 & 0.17 & -0.07 & $0 \cdot 16$ & -0.07 & 0.17 & -0.07 & 0.17 & -0.07 & 0.21 \\
\hline$\delta \mathrm{Vr} /$ & 3.00 & 0.60 & $3 \cdot 03$ & 0.52 & 2.90 & 0.58 & $2 \cdot 84$ & 0.61 & $2 \cdot 60$ & 0.56 & 2.88 & 0.46 \\
\hline $\mathrm{ms}^{-1}$ & $1 \cdot 35$ & 0.33 & $1 \cdot 34$ & 0.25 & $1 \cdot 34$ & 0.23 & $1 \cdot 34$ & 0.25 & $1 \cdot 34$ & $0 \cdot 26$ & $1 \cdot 35$ & 0.33 \\
\hline
\end{tabular}
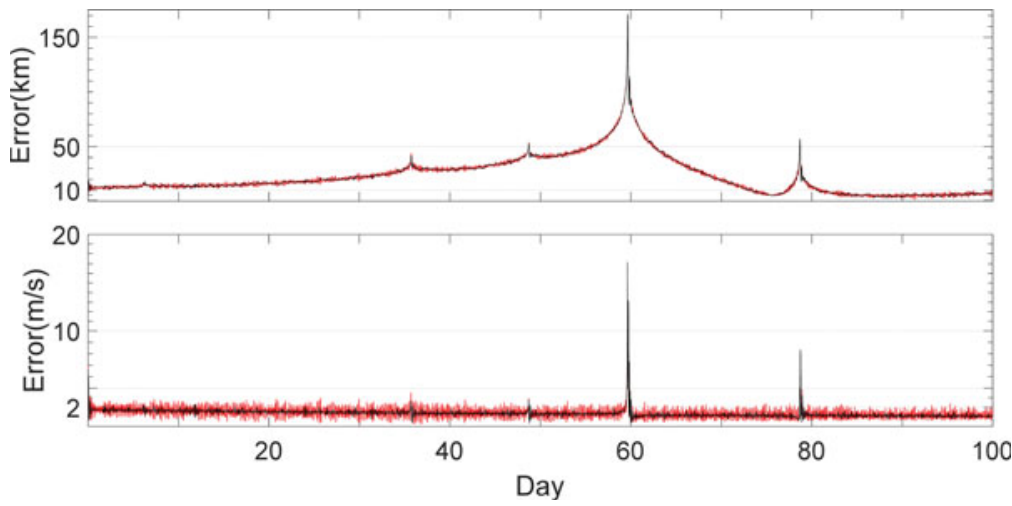

Figure 6. The systematic errors influence on orbit accuracy.

sensors still have six grades and the clock errors are the same as in the single system experiments.

Figure 6 shows the accuracy of the orbit position and velocity performance when the observations of XNAV are mixed with systematic errors in terms of Roemer Delay, Shapiro Delay and clock errors. The position bias has obviously variable tendency because the two delays have a functional relationship with the position vector of the spacecraft as shown in Equation (5). The peak near the 60th day is the orbit manoeuvring which can be detected distinctly by our filter. We have completed six experiments based on the accuracy of the celestial sensors. The best performance and worst performance are shown in Table 6 . There are no distinct differences among the uncertainties when the integration time is larger than 1000 seconds. The velocity of the spacecraft converges well except when the probe makes 
Table 6. The statistical table of the orbit accuracy under the influence of Roemer Delay and Shapiro Delay.

\begin{tabular}{|c|c|c|c|c|c|c|c|c|}
\hline \multirow{2}{*}{$\begin{array}{l}\text { Value } \\
\text { Bias }\end{array}$} & \multicolumn{4}{|c|}{ The best accuracy/1(1500 s) } & \multicolumn{4}{|c|}{ The worst $/ 6(500 \mathrm{~s})$} \\
\hline & $\max$ & $\min$ & mean & SD & $\max$ & $\min$ & mean & SD \\
\hline$\delta \mathrm{r} / \mathrm{km}$ & $171 \cdot 11$ & $3 \cdot 33$ & $23 \cdot 02$ & $17 \cdot 72$ & $170 \cdot 5$ & 1.915 & 22.99 & $17 \cdot 75$ \\
\hline$\delta \mathrm{V} / \mathrm{m} / \mathrm{s}$ & $17 \cdot 09$ & $0 \cdot 35$ & $1 \cdot 37$ & 0.49 & $16 \cdot 44$ & $0 \cdot 25$ & $1 \cdot 41$ & 0.63 \\
\hline
\end{tabular}
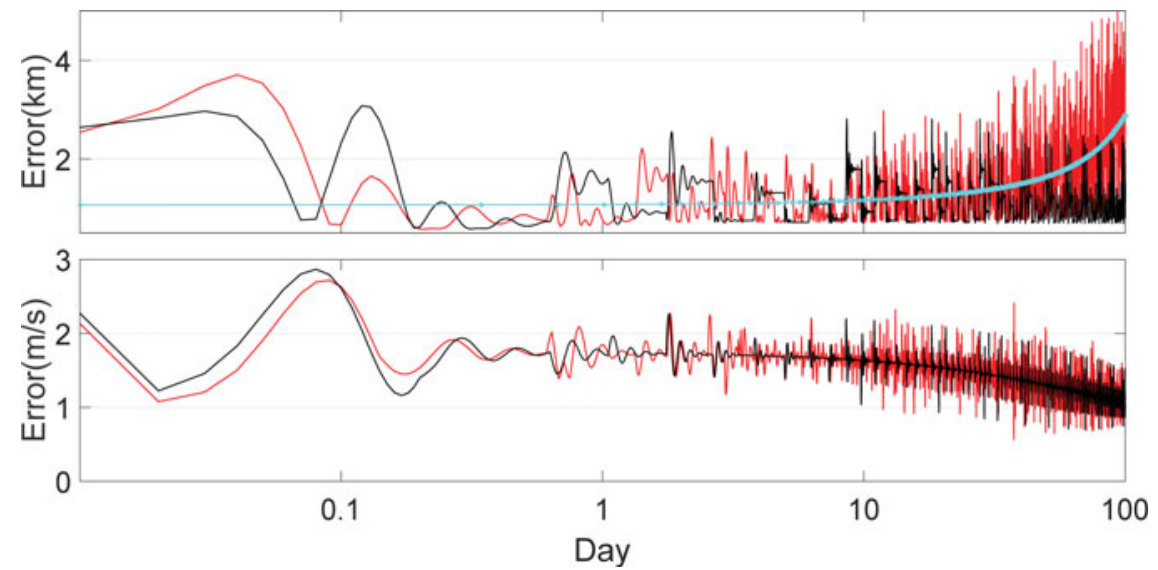

Figure 7. The orbit accuracy with clock offset equals to $10^{-7}$ seconds. The cyan arrows line is the trend of the worst position accuracy (the same in Figure 8 and Figure 9).

a manoeuvre (60th day) and the adjustment of the filter at the 80th day. The orbit is suffering a long-term drift under the influences of these two delays.

3.2.4. Experiments with different clock errors. The errors of the measurements are added in terms of celestial sensor of medium accuracy and the integration time of $1000 \mathrm{~s}$. The testing results (best and worst) are portrayed in Figures 7, 8 and 9. All of the results are shown in Table 7. The worst cases in Figures 7-9 are generated when the drift of the clock equals $10^{-8}$ seconds per day and the best cases are generated when the drift is $10^{-12}$ seconds per day. The accuracy of the orbit is stable when the clock offset is less than $10^{-8}$ seconds and clock drift less than $10^{-9}$ seconds per day. Most importantly, the worst position accuracy is within five kilometres and the worst velocity accuracy is within $4 \mathrm{~m} / \mathrm{s}$. The orbit drifts along with the drift of the clock.

3.3. Statistical evaluation of the accuracy. A general statistical evaluation of the accuracy of this paper is illustrated in Table 8 (under the condition that the Kalman Filters having converged and exclusive of orbit uncertainty caused by orbit manoeuvre). Generally, the accuracy of single OCN is very common compared with current OCN techniques that have been applied in deep space missions. However, the accuracy of single XNAV is better than the ground testing results of SEXTANT because our experiments did not take into account the orbit uncertainty caused by time-frequency transfer and profile correlation in the XNAV system.

The integrated OCN/XNAV has a good performance, which is better than the single system due to the positive feedback provided by the FKF algorithm. In addition, the accuracy of the integrated system is mostly ensured by the XNAV and the stability of the system is 

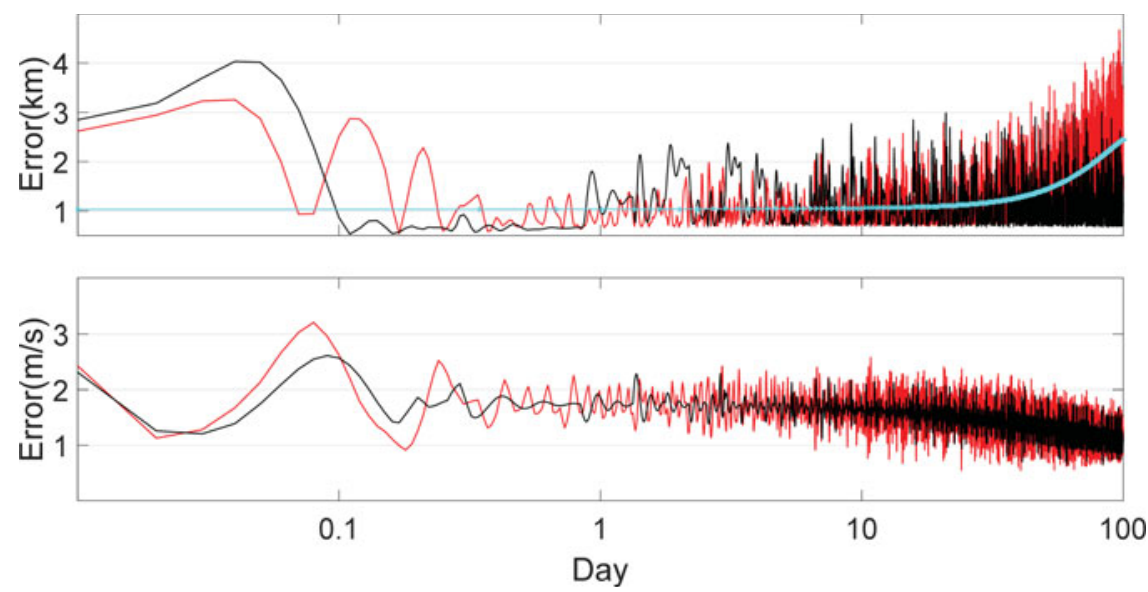

Figure 8 . The orbit accuracy with clock offset equal to $10^{-8}$ seconds.
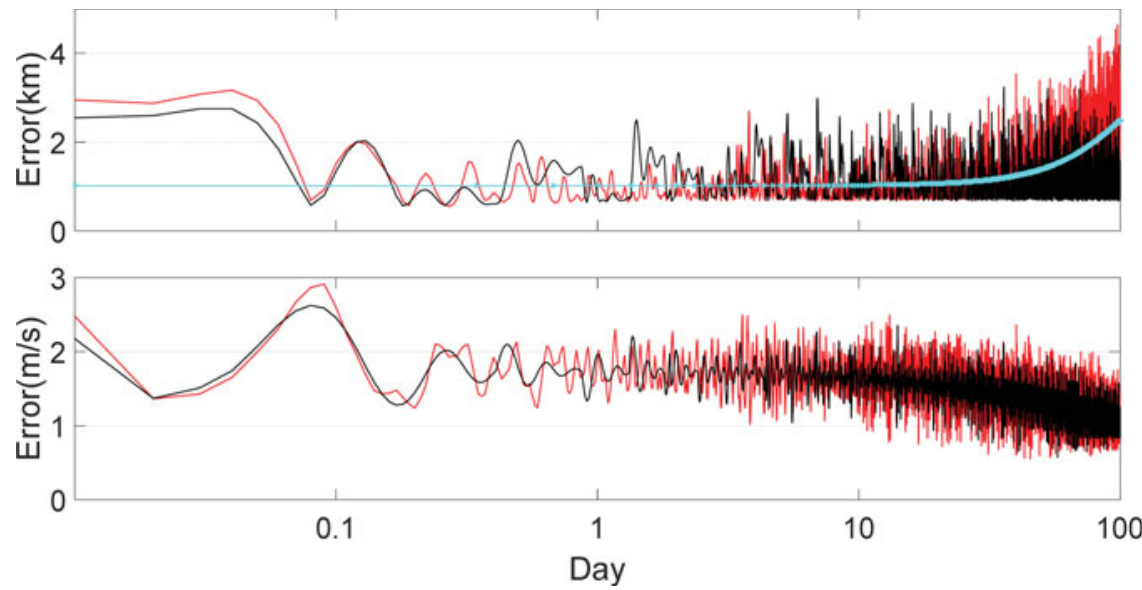

Figure 9. The orbit accuracy with clock offset equal to $10^{-9}$ seconds.

Table 7. The orbit accuracy with different clock errors.

\begin{tabular}{|c|c|c|c|c|c|c|c|c|c|c|c|c|c|}
\hline \multirow{2}{*}{$\begin{array}{c}\mathrm{a}_{1} \\
\mathrm{a}_{0} \text { (second/day) }\end{array}$} & & \multicolumn{3}{|c|}{$10^{-7} \mathrm{~s}$} & \multicolumn{4}{|c|}{$10^{-8} \mathrm{~s}$} & \multicolumn{4}{|c|}{$10^{-9} \mathrm{~s}$} & \multirow[b]{2}{*}{ SD } \\
\hline & & $\max$ & $\min$ & mean & SD & Max & $\min$ & mean & SD & Max & $\min$ & mean & \\
\hline \multirow[t]{2}{*}{$10^{-} 8$} & $\delta \mathrm{r} / \mathrm{km}$ & $5 \cdot 00$ & 0.58 & 1.74 & 0.86 & $4 \cdot 67$ & 0.54 & 1.54 & 0.70 & 4.64 & 0.55 & 1.52 & 0.71 \\
\hline & $\delta \mathrm{V} / \mathrm{ms}^{-} 1$ & $2 \cdot 72$ & 0.57 & 1.34 & 0.26 & $3 \cdot 21$ & 0.55 & $1 \cdot 35$ & 0.34 & 3.92 & 0.55 & $1 \cdot 35$ & $0 \cdot 33$ \\
\hline \multirow[t]{2}{*}{$10^{-9}$} & $\delta \mathrm{r} / \mathrm{km}$ & $4 \cdot 51$ & 0.57 & $1 \cdot 35$ & 0.56 & $3 \cdot 95$ & 0.57 & $1 \cdot 13$ & 0.42 & 3.66 & 0.55 & 1.08 & 0.40 \\
\hline & $\delta \mathrm{V} / \mathrm{ms}^{-1}$ & $3 \cdot 20$ & 0.57 & $1 \cdot 36$ & 0.38 & 3.02 & 0.59 & $1 \cdot 34$ & 0.25 & $2 \cdot 83$ & 0.52 & $1 \cdot 35$ & $0 \cdot 36$ \\
\hline \multirow[t]{2}{*}{$10^{-10}$} & $\delta \mathrm{r} / \mathrm{km}$ & $4 \cdot 36$ & 0.55 & 1.09 & 0.39 & $3 \cdot 10$ & 0.54 & $1 \cdot 11$ & 0.43 & $3 \cdot 24$ & 0.56 & $1 \cdot 19$ & 0.46 \\
\hline & $\delta \mathrm{V} / \mathrm{ms}^{-1}$ & $2 \cdot 78$ & 0.57 & $1 \cdot 35$ & 0.29 & $2 \cdot 73$ & 0.49 & 1.35 & 0.36 & 2.63 & 0.57 & $1 \cdot 34$ & $0 \cdot 26$ \\
\hline \multirow[t]{2}{*}{$10^{-11}$} & $\delta \mathrm{r} / \mathrm{km}$ & $3 \cdot 59$ & 0.57 & $1 \cdot 15$ & 0.42 & $3 \cdot 64$ & 0.58 & $1 \cdot 20$ & 0.47 & $3 \cdot 24$ & 0.56 & $1 \cdot 19$ & 0.46 \\
\hline & $\delta \mathrm{V} / \mathrm{ms}^{-1}$ & $2 \cdot 82$ & 0.67 & $1 \cdot 34$ & 0.25 & $2 \cdot 86$ & 0.58 & $1 \cdot 34$ & 0.27 & 2.63 & 0.57 & $1 \cdot 34$ & $0 \cdot 26$ \\
\hline \multirow[t]{2}{*}{$10^{-12}$} & $\delta \mathrm{r} / \mathrm{km}$ & $3 \cdot 08$ & 0.58 & $1 \cdot 10$ & 0.34 & 4.03 & 0.53 & $1 \cdot 15$ & 0.44 & $3 \cdot 24$ & 0.56 & $1 \cdot 19$ & 0.46 \\
\hline & $\delta \mathrm{V} / \mathrm{ms}^{-1}$ & $2 \cdot 87$ & 0.70 & $1 \cdot 34$ & 0.22 & $2 \cdot 61$ & 0.64 & $1 \cdot 34$ & 0.25 & 2.63 & 0.57 & $1 \cdot 34$ & $0 \cdot 26$ \\
\hline
\end{tabular}


Table 8. The general statistic of the accuracy of OCN, XNAV and integrated OCN/XNAV (absolute value).

\begin{tabular}{|c|c|c|c|c|c|c|}
\hline \multirow[b]{2}{*}{ System } & \multicolumn{3}{|c|}{ 3-axis position bias $(\mathrm{km})$} & \multicolumn{3}{|c|}{3 -axis velocity bias $(\mathrm{m} / \mathrm{s})$} \\
\hline & $\max$ & $\min$ & mean & $\max$ & $\min$ & mean \\
\hline$O C N$ & 140 & 100 & 120 & 130 & 100 & 120 \\
\hline$X N A V$ & $15 \cdot 0$ & 0 & $2 \cdot 5$ & 25 & 10 & 16 \\
\hline$O C N / X N A V$ & $5 \cdot 0$ & 0 & $2 \cdot 5$ & $4 \cdot 0$ & $0 \cdot 0$ & $2 \cdot 0$ \\
\hline
\end{tabular}

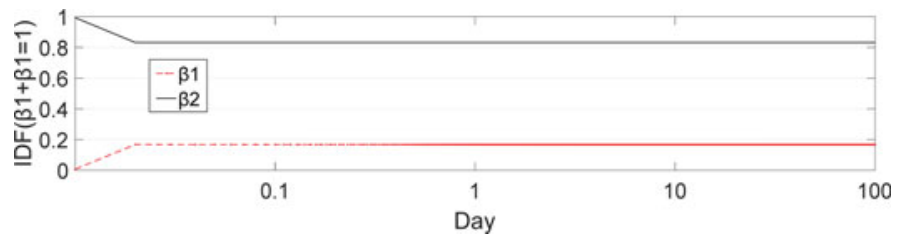

Figure 10. The IDF of OCN system $(\beta 1)$ and XNAV system $(\beta 2)$ (General case).
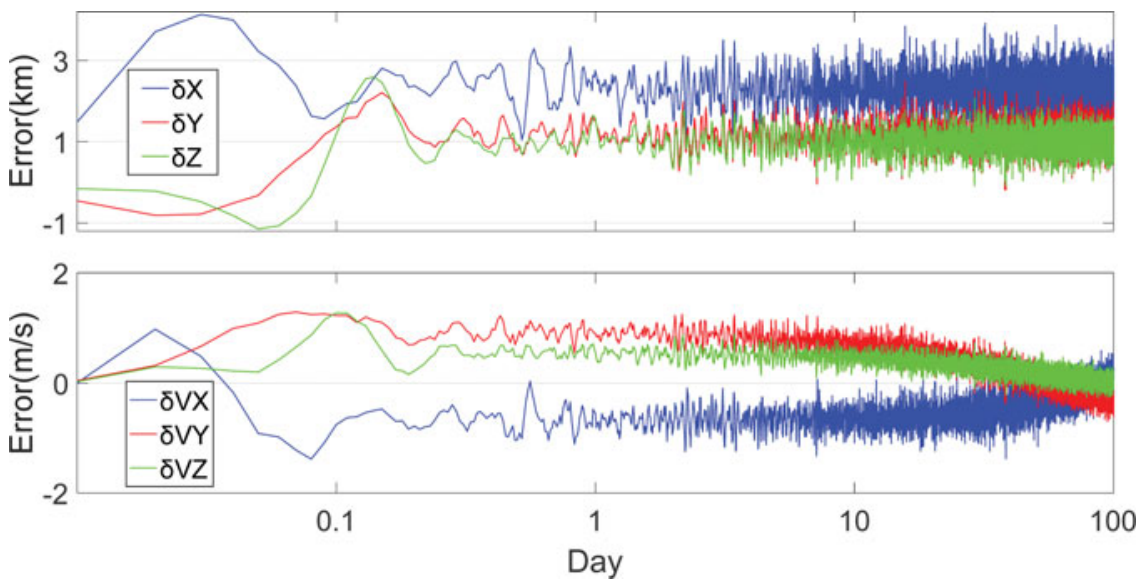

Figure 11. The performance of OCN subsystem after the feedback adjustment (General case).

mostly ensured by the OCN system. The ratio of IDF of OCN and IDF of XNAV is about 0.18:0.82 (Figure 10), which shows that the accuracy of the integrated system is mostly provided by XNAV. At the same time, the subsystem of OCN inherits the accuracy of the main system completely as illustrated in Figure 11.

The accuracy of the XNAV subsystem is more stable than the single XNAV and the accuracy converged very well as portrayed in Figure 12. In other words, the OCN system has a very strong influence on the stability performance of the integrated system.

However, a few drawbacks do exist in the system. The potential disadvantages of our system might be as follows: The observational platform of OCN might be so large that the spacecraft cannot use this hardware load. This problem can be solved by using a narrowfield navigation camera such as the one on board MRO. Secondly, the number of observable $\mathrm{X}$-ray Pulsars might be too many to scan. The limited number of observations will influence the stability of the XNAV. 

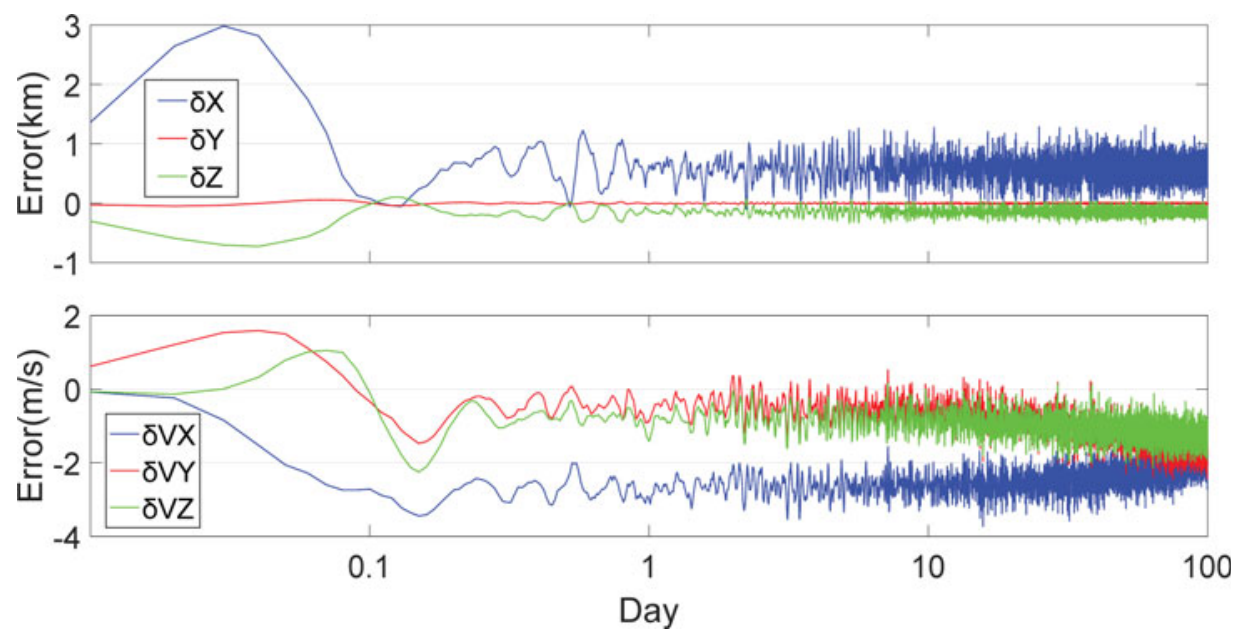

Figure 12. The accuracy performance of XNAV subsystem after the feedback adjustment (General case).

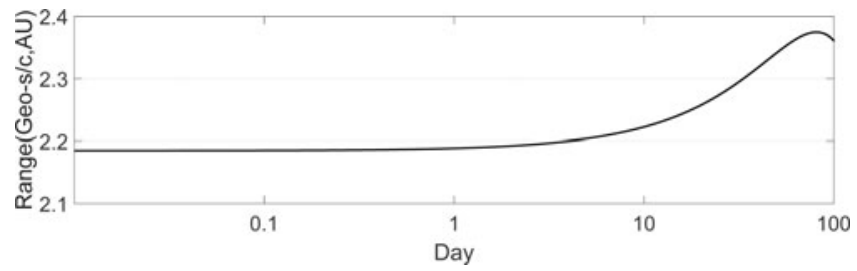

Figure 13. The Geocentric range of Mars Pathfinder spacecraft $(\mathrm{s} / \mathrm{c})$ in this paper.

3.4. Comparison with current ground tracking techniques. The state-of-the-art ground-based tracking techniques or observations utilised in deep space navigation are radiometric observational methods in terms of two-way Doppler, two-way range and Delta-DOR (Bhaskaran, 2012). Traditionally, two-way Doppler and range observations are acquired by ground radar in the $\mathrm{S} / \mathrm{X}$ band, which is also known as Unified S-Band (USB). The Delta-DOR use VLBI antennas to observe the Quasar and the signal broadcast by the on board signal source.

The accuracy of time delay of Delta-DOR is about one nanosecond which corresponds to an orbit accuracy of four kilometres per AU (James et al., 2009). The Geocentric range of spacecraft in this paper is larger than two AU (Figure 13), that is, if we use current high accuracy Delta-DOR to navigate the probe then the orbit uncertainty would not be better than eight kilometres.

4. CONCLUSION. In this paper, an integrated Optical Celestial Navigation (OCN) and X-ray Pulsars Autonomous Navigation (XNAV) system is realised and evaluated for the orbit of Mars Pathfinder. Our autonomous navigation system can provide a stable and high accuracy orbit solution for a Mars probe or other deep space spacecraft. The performance of the system is better than single OCN and XNAV. Firstly, the system can acquire an accuracy of position/velocity within five kilometres and $4 \mathrm{~m} / \mathrm{s}$ even though the uncertainty of the celestial sensors is large and the XNAV signal process time is short. Secondly, the Roemer 
delay and Shapiro delay have a major influence on the orbit accuracy. Thus, these kinds of systematic errors of the system should be modelled or eliminated before the navigation. Thirdly, if the offset and drift of the clock are better than $10^{-9}$ seconds and $10^{-9}$ seconds per day respectively, these terms have limited or minor influence on navigational accuracy. Otherwise, these errors should be taken into account to obtain a precise orbit. The system's performances such as accuracy and autonomous ability are better than current ground-based tracking methods.

\section{ACKNOWLEDGMENTS}

This work was supported by the National Keystone Basic Research Program (MOST 973) (Grant no. 2012CB72000), National Natural Science Foundation of China (No. 41374012, 11173050 and 11373059), and Shanghai Science and Technology Commission Project (No. 12DZ2273300).

\section{REFERENCES}

Becker, W., Bernhardt, M.G. and Jessner, A. (2013). Autonomous Spacecraft Navigation With Pulsars. Acta Futura, 7, 11-28.

Bhaskaran, S. (2012). Autonomous navigation for deep space missions. SpaceOps 2012 Conference, Stockholm, Sweden.

Cao, J.F., Yong, H., Hu, X.G., Ma, M.L. and Zheng, W.M. (2010). Mars express tracking and orbit determination trials with chinese vlbi network. Chinese Science Bulletin, 55(32), 3654-3660.

Carlson, N.A. (1990). Federated square root filter for decentralized parallel processors. IEEE Transactions on Aerospace and Electronic Systems, 26(3), 517-525.

Chester, T.J. and Butman, S.A. (1981). Navigation using X-ray pulsars. NASA technique report 81N27129, $22-25$.

Deng, X.P., Hobbs, G., You, X.P., Li, M.T., Keith, M.J., Shannon, R.M., Coles, W., Manchester, R.N., Zheng, J.H., Yu, X.Z., Gao, D., Wu, X. and Chen, D. (2013). Interplanetary spacecraft navigation using pulsars. Advances in Space Research, 52(9), 1602-1621.

Dormand, J.R. and Prince, P.J. (1978). New Runge-Kutta algorithms for numerical simulation in dynamical astronomy. Celestial Mechanics, 18(3), 223-232.

Downs, G.S. (1974). Interplanetary navigation using pulsating radio sources. NASA technique report 32-1954, 1-10.

Golombek, M.P., Cook, R.A., Economou, T., Folkner, W.M., Haldemann, A.F., Kallemeyn, P.H., Knudsen, J.M., Manning, R.M., Moore, H.J., Parker, T.J., Rieder, R., Schofield, J.T., Smith, P.H. and Vaughan, R.M. (1997). Overview of the Mars Pathfinder mission and assessment of landing site predictions. Science, 278(5344), 17431748 .

Gounley, R., White, R. and Gai, E. (1984). Autonomous satellite navigation by stellar refraction. Journal of guidance, control, and dynamics, 7(2), 129-134.

Graf, J.E., Zurek, R.W., Eisen, H.J., Jai, B. Johnstona, M.D. and Ramon, DePaulab. (2005). The Mars reconnaissance orbiter mission. Acta Astronautica, 57(2), 566-578.

Graven, P., Collins, J., Sheikh, S., Hanson, J., Ray, P., and Wood, K. (2008). XNAV for deep space navigation. 31st Annual AAS Guidance and Control Conference, AAS 08-054, 1-16.

Hampton, D.L., Baer, J.W., Huisjen, M.A., Varner, C.C., Delamere, A., Wellnitz, D.D., A'Hearn, M.F. and Klaasen, K.P. (2005). An overview of the instrument suite for the Deep Impact mission. Space Science Reviews, 117(1-2), 43-93.

Hanson, J.E. (2006). Principles of X-ray Navigation. SLAC Report 809, Chapter 1, 1-6.

Hemmati, H. (2006). Deep space optical communications. John Wiley and Sons, 11, Chapter1, 1-5.

James, N., Abello, R., Lanucara, M., Mercolino, M., and Maddè, R. (2009). Implementation of an ESA Delta-DOR capability. Acta Astronautica, 64(11), 1041-1049.

Jin, S.G., Arivazhagan, S., and Araki, H. (2013). New results and questions of lunar exploration from SELENE, Chang'E-1, Chandrayaan-1 and LRO/LCROSS, Advances in Space Research, 52(2), 285-305.

Jin, S.G. and Zhang, T.Y. (2014). Automatic detection of impact craters on Mars using a modified adaboosting method, Planetary and Space Science, 99, 112-117, doi: 10.1016/j.pss.2014.04.021. 
Lowman, A.E. and Stauder, J.L. (2004). Stray light lessons learned from the Mars Reconnaissance Orbiter's Optical Navigation Camera. Proceedings of SPIE 5526, 5526, 240-248.

Mastrodemos, N., Kubitschek, D.G. and Synnott, S.P. (2005). Autonomous navigation for the Deep Impact mission encounter with comet Tempel 1. Space Science Reviews, 117(1-2), 95-121.

Rong, J., Luping, X., Zhang, H. and Li, C. (2016). Augmentation method of XPNAV in Mars Orbit based on Phobos and Deimos observations. Advances in Space Research.

Sheikh, S.I. and Pines, D.J. (2006). Recursive Estimation of Spacecraft Position and Velocity Using X-ray Pulsar Time of Arrival Measurements. Navigation, 53(3), 149-166.

Sheikh, S.I., Hellings, R.W. and Matzner, R.A. (2007). High-order pulsar timing for navigation. Proceedings of the $63^{\text {rd }}$ ION Annual Meeting, Cambridge, Massachusetts, 432-443.

Sheikh, S.I., Pines, D.J., Ray, P.S., Wood, K.S., Lovellette, M.N., and Wolff, M.T. (2006). Spacecraft navigation using X-ray pulsars. Journal of Guidance, Control, and Dynamics, 29(1), 49-63.

Sierks, H., Keller, H.U., Jaumann, R., Michalik, H., Behnke, T., Bubenhagen, F., Büttner, I., Carsenty, U., Christensen, U., Enge, R., Fiethe, B., Gutiérrez, M.P., Hartwig, H., Krüger, H., Kühne, W., Maue, T., Mottola, S., Nathues, A., Reiche, K.U., Richards, M.L., Roatsch, T., Schröder, S.E., Szemerey and Tschentscher, I.M. (2011). The Dawn framing camera. Space science reviews, 163(1-4), 263-327.

Soderblom, L.A., Becker, T.L., Bennett, G., Boice, D.C., Britt, D.T., Brown, R.H., Buratti, B.J., Isbell, C., Giese, B., Hare, T., Hicks, M.D., Howington-Kraus, E., Kirk, R.L., Lee, M., Nelson, R.M., Oberst, J., Owen, T.C., Rayman, M.D., Sandel, B.R., Stern, S.A., Thomas, N. and Yelle, R.V. (2002). Observations of Comet 19P/Borrelly by the miniature integrated camera and spectrometer aboard Deep Space 1. Science, 296(5570), 1087-1091.

Stastny, N.B. and Geller, D.K. (2008). Autonomous optical navigation at Jupiter: a linear covariance analysis. Journal of Spacecraft and Rockets, 45(2), 290-298.

Sun, T., Xing, F., Wang, X., You, Z., and Chu, D. (2016). An accuracy measurement method for star trackers based on direct astronomic observation. Scientific reports, 2016, 6(22593):1-10.

Wan, E.A., and Van der Merwe, R. (2000). The unscented Kalman filter for nonlinear estimation. AS-SPCC. IEEE $153-158$

Wang, Y., Zheng, W., An, X., Sun, S. and Li, L. (2013). XNAV/CNS integrated navigation based on improved kinematic and static filter. Journal of Navigation, 66(6), 899-918.

Wei, E., Jin, S., Zhang, Q., Liu, J., Li, X. and Yan, W. (2013). Autonomous navigation of Mars probe using X-ray pulsars: modeling and results. Advances in Space Research, 51(5), 849-857.

Wei, E., S. Tang, S.G. Jin, and J. Liu (2016). Positioning results of lunar rover based on combined VLBI and Celestial Navigation, Journal of Geodesy and Geodynmics, 36(8), 703-707.

White, R.L., Thurman, S.W. and Barnes, F.A. (1985). Autonomous satellite navigation using observations of starlight atmospheric refraction. Navigation, 32(4), 317-333.

Winternitz, L.M.B., Mitchell, J.W., Hassouneh, M.A., Valdez, J.E., Price, S.R., Semper, S.R. and Yu, W.H. (2016). SEXTANT X-Ray Pulsar Navigation Demonstration: Flight System and Test Results. NASA technique report $20160003320,1-12$. 